\title{
LA FILOSOFÍA DEL LENGUAJE Y LA SEMIÓTICA. PROLEGÓMENOS
}

\author{
Mariana Neţ \\ Instituto de Lingüística «Iorgu Iordan» (Bucarest)
}

A primera vista, la filosofía del lenguaje aparece como una disciplina fronteriza, situada en el cruce entre la lingüística teórica y la filosofía.

Quizás, precisamente por eso, sea difícil de definir. La multitud casi infinita de escuelas y corrientes lingüísticas y filosóficas, su diseminación en espacio y tiempo - como bien se sabe, podríamos empezar, arbitraria y convencionalmente, ese panorama con Platón y podríamos ponerle un punto final, igual de arbitrario y provisional, tomando en cuenta los textos de Noam Chomsky (1986)_, parece destinada a desanimar al investigador que se atreviera a acercarse a este ámbito. Además, las dificultades a las que se enfrenta el que se ha propuesto un objetivo tan ambicioso son, generalmente, mayores - y no menores- dada la multitud sin fin de síntesis y de aclaraciones - muchas veces divergentes - que ha aportado el último siglo. Siguiendo el mismo razonamiento, hay que recordar también que en los últimos cien años se han puesto - explícitamente — las bases de otros ámbitos 
inter-y trans-disciplinares, como, por ejemplo, la filosofía de la historia, la filosofía de la religión, la filosofía de la ciencia, la filosofía política, la sociolingüística, la psicolingüística, etc.

Estos fenómenos complican aún más la tarea — difícil de por sídel investigador. Dado que las ciencias ya no se pueden considerar por separado - la atomización que ha caracterizado, en gran parte, el siglo XIX debe ser sustituida por el retorno a una visión globalizadora, parecida a la que ha dominado en la Edad Media y el Renacimiento- el investigador debe afilar sus utensilios permanentemente y establecer con toda claridad un punto de partida. La flexibilidad y la constancia no están reñidas.

Todo esto proporciona un argumento a favor de la hipótesis según la cual el que se propone tratar el complejo ámbito de la filosofía del lenguaje debería partir de cero (o asumir que lo hace). Es decir, debe empezar por establecer claramente su ámbito y sus instrumentos de trabajo. Precisamente porque todo es dado, nada es a priori.

Con este objetivo, vamos a presentar a continuación los resultados de un recorrido eurístico, que se ha propuesto delimitar poco a poco el campo complejo de la filosofía del lenguaje, más precisamente establecer aquellos sectores de la disciplina mencionada que vamos a desarrollar en una serie de estudios ulteriores.

Ya que nuestra perspectiva va a ser predominantemente semiótica - he aquí ya una opción metodológica-, nos parece normal empezar por tomar en consideración el modo en el que se relacionan los semióticos con el ámbito mencionado.

Una primera observación que se impone - y que nos debería hacer pensar- es que no hay ningún artículo dedicado a ese ámbito en los volúmenes del Diccionario de Greimas y Courtès $(1979,1986)$, como tampoco en el Glosario de términos semióticos de Vincent Colapietro (1993).

En cambio, el Encyclopedic Dictionary of Semiotics (1986) -que citaré como $E D S$ - le dedica a esta disciplina un amplio artículo de catorce páginas (1986: 708-721). Hacia el final del artículo (p. 714), después de pasar revista a las principales acepciones de la disciplina, los autores del EDS apuntan: «[ya que] la semiótica toma en consideración, entre otros, los aspectos filosóficos de los signos y los problemas filosóficos relacionados con los signos [...], se puede considerar que la filosofía del lenguaje constituye una parte de la semiótica». 
Antes de llegar a esta conclusión — previsible—, los autores de la obra monumental arriba mencionada empiezan sin embargo, y con razón, creemos, por precisar (pp. 708-711) que la esfera de la filosofía del lenguaje depende de la manera en que comprendemos los términos filosofía y lenguaje, siendo estas palabras «entre las más polisemánticas, ambiguas y abstractas de todas las ciencias humanistas» (p. 709). He aquí por qué, prosiguen, el concepto de filosofía del lenguaje es tributario de las deficiencias de los dos términos que constituyen el sintagma que lo designa.

Sólo el término filosofía tiene, al menos, seis sentidos ${ }^{1}$ distintos. Vayamos por partes ${ }^{2}$ :

1) Según la primera acepción, el sustantivo filosofía denota una colección heterogénea de temas enseñados por las cátedras universitarias que llevan este nombre. La metafísica, la epistemología, la ética, la estética, la historia de la filosofía, la psicología, la filosofía de la ciencia, la filosofía de la historia y la filosofía de la religión. «A lo largo del tiempo, las últimas cuatro disciplinas han llegado a no pertenecer ya a la filosofía» (Sebeok, 1986: 709).

Se imponen algunos comentarios con respecto a este punto, desde la perspectiva que aquí nos interesa:

a) El hecho de que la psicología haya sido enseñada en las cátedras de filosofía de los Estados Unidos (y no sólo ahí) parece extraño, aún en el caso en el que se tratara de una división estrictamente administrativa. No debemos olvidar, sin embargo, que en algunos de los últimos trabajos de filosofía del lenguaje elaborados por Noam Chomsky (1986), la psicología juega un papel bastante importante en su teoría sobre la evolución y las funciones de la lengua en la sociedad. Este aspecto quedará sin embargo, en cuanto lo sea posible, fuera de nuestras futuras preocupaciones.

b) Aunque es verdad que la filosofía de la ciencia, la filosofía de la historia y la filosofía de la religión se han convertido, con el tiempo,

1 Para no complicar más las cosas con una discusión sobre las diferencias entre significado y significación, hemos optado aquí por el término de «sentido» (înteles en el original rumano).

${ }^{2}$ Se sobreentiende que presentamos aquí sólo una síntesis parcial de algunos capítulos dedicados al término «filosofía del lenguaje» del Enciclopedic Dictionary of Semiotics (1986: 708-721). 
en disciplinas autónomas, se sitúan ahora, al igual que la filosofía del lenguaje, en el ámbito de la inter- y la trans-disciplinaridad; sus relaciones con la filosofía del lenguaje - como, entre otras, también con el ámbito más amplio de la semiótica — son bastante importantes para merecer ser tratadas en un estudio ulterior.

c) La metafísica y la ética se pueden excluir (por lo menos temporalmente) de las preocupaciones de la filosofía del lenguaje. No se puede decir lo mismo de la lógica formal, que se entrelaza - a menudo hasta la superposición - con múltiples preocupaciones de la filosofía del lenguaje, sobre todo en la segunda mitad del siglo XX (cf. inter alii Carnap, 1960; Kalish-Montague, 1964; Hintikka, 1969). A pesar de todo esto, tal orientación epistémica y metodológica se va a quedar fuera de nuestras futuras preocupaciones.

d) Totalmente diferentes se ven las cosas en cuanto a la epistemología y la estética. La primera de estas disciplinas depende de la esencia misma de la filosofía del lenguaje; la relación entre los dos ámbitos debe ser desarrollada, por eso, en una serie de estudios ulteriores. A su vez, la estética tiene estrechas relaciones, aunque indirectas, con el tema que nos preocupa aquí, si le proporcionamos a la estética una acepción más amplia, que se interrelaciona con la retórica y con la poética.

2) La segunda acepción del Encyclopedic Dictionary of Semiotics no nos va a interesar en estas páginas, ya que se refiere exclusivamente a la presencia del término filosofía en la denominación de unas cátedras universitarias.

3) La tercera acepción del EDS (1986: 709) concierne a la filosofía vs. la ciencia, considerando que «la primera constituye un Weltanschauung general [...], basado en datos proporcionados por otras disciplinas; estos datos se refieren a problemas como la causalidad [...], la muerte y la pérdida de la conciencia [...]. Se refiere, también, a una serie de relaciones como la que hay entre conocimiento, por un lado, y cosas y fenómenos, por otro lado; la que hay entre valores, por un lado, y cosas, por otro lado, al igual que a la relación entre el universo y su creador».

Este punto tiene especial importancia para el estudio de la filosofía del lenguaje:

a) Primero, porque esta disciplina incluye prioritariamente reflexiones sobre la relación de causalidad en la esfera de la lengua y de los 
lenguajes, la modalidad en la que la lengua y los lenguajes contribuyen al conocimiento de las cosas y de los fenómenos —el hecho de que la epistemología sea una parte importante de la filosofía del lenguaje se pone de nuevo en evidencia - y sobre la manera en la que la lengua y los lenguajes confieren «valor» a las cosas, gracias al acto de denominación y a la integración en contextos. Desde este punto de vista, el ámbito del que nos ocupamos aquí se acerca a otras disciplinas aparentemente fronterizas con la lingüística, como, por ejemplo, la pragmática, la retórica y la poética.

b) En cuanto a la relación entre el universo y su creador, la discusión parece infinita, y los matices entre el provecho máximo y la esterilidad son múltiples y varían en el tiempo en función de una multitud de factores que no vamos a estudiar. Bastará recordar, de paso, la multitud de debates referentes a la equivalencia o a las diferencias entre logos y verbum, según la traducción griega o latina del célebre incipit del Evangelio según San Juan (I, 1): «Primero fue la Palabra y la Palabra era de Dios y Dios era la Palabra» (1975: 1197). En función de una o de otra de las distintas opciones con las que se encuentra el filólogo, toda una concepción del mundo modifica sus matices de manera significativa.

c) A la luz de todo lo anterior, el estudio de los diccionarios - de los explicativos, pero también de los enciclopédicos - adquiere importancia para el ámbito en cuestión.

4) Según la cuarta acepción del término «filosofía» que registra el Encyclopedic Dictionary of Semiotics, el concepto designa, también, «una etapa pre-científica en el desarrollo de cierta disciplina» (1986: 709).

Consideramos necesaria una serie de observaciones referentes a este punto:

Aún cuando «pre-científico» no sea, quizás, el calificativo más adecuado, parece evidente que, de acuerdo con la acepción arriba mencionada, la esfera de la filosofía del lenguaje abarca estudios referentes a la puesta en texto y en discurso, a la formulación de las concepciones sobre el mundo que implica cualquier disciplina, a la relación de las ciencias y del sujeto conocedor con el mundo, etc. Todo esto incluye elementos -incluso rudimentarios- de filosofía del lenguaje.

Una inferencia similar aparece también en un antiguo estudio de hace aproximadamente dos decenios de Thomas A. Sebeok, que con- 
sidera que «las preconcepciones filosóficas determinan las preferencias terminológicas» (Sebeok, 1981: 11).

De todas maneras, en una formulación diferente, el principio se reencuentra también en el siguiente punto del Encyclopedic Dictionary of Semiotics (1986: 709).

5) «El término filosofía, contenido en expresiones como filosofía de cierta ciencia, designa la teoría de esta ciencia $y$, por consiguiente, un análisis crítico meta-científico de los conceptos y de los métodos empleados por ésta».

Esta vez también consideramos necesaria una serie de matizaciones:

a) Desde la perspectiva que hemos adoptado aquí, esta acepción implica (si no la equivalencia, en todo caso) la perfecta compatibilidad entre la filosofía del lenguaje y la linguiística teórica, sobre todo en la hipóstasis de esta última como estudio de los metalenguajes.

b) A su vez, el estudio de los metalenguajes puede ser abarcado desde una multitud de perspectivas: lógico-semántica, retórica, pragmática. La última nos parece, con diferencia, la más auténtica. Naturalmente, otros investigadores pueden optar por una metodología diferente.

c) El estudio de los metalenguajes es de la competencia de la semiótica lingüística e incluso de la semiótica en general. La relación de compatibilidad entre la filosofía del lenguaje y la semiótica se muestra de esta manera, de nuevo, evidente, mientras que una estricta separación entre las dos perspectivas nos parece irrelevante y estéril.

d) El metalenguaje del texto literario ocupa un lugar importante en el estudio de los metalenguajes como métodos pertenecientes a la lingüística teórica, a la filosofía del lenguaje y a la semiótica. En este caso, la última se constituye en una (meta)retórica.

e) Si el metalenguaje del texto literario está incluido en el texto, es decir, pertenece a una hipóstasis del Enunciador, el discurso literario cumple una función autonómica, que merece ser objeto de estudios independientes. Al mismo tiempo, se tiene que señalar que, en casos así, el discurso literario tiende, como se ha mostrado en otras ocasiones (Net, 1989), a convertirse en otra clase o subclase de discurso. 
f) «El análisis crítico, meta-científico» (cf. supra) de la linguiística nos devuelve a la lingüística teórica, disciplina que, a su vez, se vuelve de nuevo equivalente de la filosofía del lenguaje, tal y como ésta se ha entendido en las presentes páginas.

Volvamos al Diccionario del que partimos y pasemos en revista la última de las acepciones que sus autores le confieren al término de filosofía:

6) Se trata de la «filosofía como ciencia de la ciencia en general, y no como una ciencia dedicada a cierta ciencia» (1989: 709).

Como era de esperar, también en este punto se impone una serie de observaciones y matizaciones:

a) Si consideramos la filosofía como una «ciencia de la ciencia en general», implícitamente será una ciencia de las ciencias, y sus relaciones con la teología patrística aparecerán como evidentes. Para la filosofía del lenguaje, subrayar esta relación puede resultar muy rentable. El hecho de que padres de la Iglesia como San Agustín (1994), para mencionar sólo el caso más conocido, trata este ámbito, al que le aporta importantes contribuciones, al igual que la constatación que las escrituras de este tipo son reivindicadas, en igual medida, por especialistas en teología, en filosofía, en semiótica y en lingüística, dejarán de sorprendernos.

b) Desde la misma perspectiva, nos resultará fácil comprender la aparición de disciplinas fronterizas como la filosofía de la historia, la filosofía de la ciencia (incluyendo aquí también la filosofía política, en la medida en que la política es una ciencia), la filosofía de la religión $y$, por supuesto, la filosofía del lenguaje, de la que ninguna de las disciplinas mencionadas puede prescindir, ya que a menudo toman prestada su metodología.

c) Así, la revisión de las acepciones implícitas o explícitas del término filosofía en el Encyclopedic Dictionary of Semiotics se cierra circularmente, aunque el concepto como tal - y la disciplina, mucho más - sigue heterogéneo.

Esta corta revisión - necesariamente incompleta y casi tautológica- lleva a las siguientes conclusiones provisionales: 
a) La lengua y los lenguajes confieren «valor» a las cosas gracias al acto de denominación y a la integración en contextos.

b) La filosofía del lenguaje abarca también un conjunto de conocimientos sobre el mundo, siendo, al mismo tiempo, una epistemología.

c) La filosofía del lenguaje se interrelaciona con la filosofía de la historia, la filosofía de la ciencia y la filosofía de la religión; todo esto constituye un argumento de más a favor del estudio de las mentalidades desde la perspectiva de la filosofía del lenguaje.

d) La filosofía del lenguaje se interrelaciona con la retórica, la pragmática y la poética en cuanto disciplinas de la lingüística.

e) La filosofía del lenguaje se interrelaciona con la lingüística teórica; las dos disciplinas son perfectamente compatibles $\mathrm{y}$, muchas veces, llegan incluso a identificarse.

f) Toda filosofía del lenguaje tiene un metalenguaje específico. Por otra parte, la filosofía del lenguaje implica el estudio de los metalenguajes (por supuesto, tanto la poética como la retórica tienen como objetivo el estudio de los metalenguajes, pero, en el caso de estas últimas disciplinas, se trata exclusivamente -o prioritariamente- del estudio del metalenguaje del texto literario).

g) Desde la perspectiva de la filosofía del lenguaje, la semiótica es (también) una (meta)retórica.

La segunda sección del presente artículo se basa en una definición del lenguaje dada, hace más de un decenio y medio, por Emanuel Vasiliu (1984: 56), quien ha demostrado que «[un lenguaje es] un fragmento de lengua natural».

La distinción entre lengua natural y lenguajes aparece, de todas formas, en la mayoría de los trabajos teóricos dedicados a una u otra de estas dos categorías. De este modo, en el primer volumen del Diccionario de Greimas y Courtès (1979: 203-204) —al que, a continuación, vamos a hacer referencia llamándolo Sémiotique-el lenguaje, en sentido general, es denominado también «lenguaje semiótico»o «conjunto significante» y se opone explícitamente a «la lengua natural». En cuanto a esta última, en el diccionario enciclopédico Filosofía de A a Z ([1994] 1999: 298) —desde ahora en adelante, $A-Z$ - la lengua aparece con «[el] significado [común]: producto social de la facultad del lenguaje, conjunto de convenciones necesarias adaptadas por el corpus 
social para permitir a los individuos ejercer esta función (ej., la lengua inglesa)».

Se pone así de manifiesto, sin lugar a duda, la dimensión social de la lengua natural. Esta dimensión nos va a interesar, de manera primordial, también en el caso del lenguaje.

Por otra parte, también en el A-Z ([1994]1999: 299) se pone el acento en la dimensión semiótica del lenguaje; en este sentido, los autores distinguen «entre lenguaje, como facultad o aptitud de constituir un sistema de signos, y lengua, que es el instrumento de comunicación propio de una comunidad humana: una lengua representa un conjunto instituido y estable de símbolos verbales o escritos, propio de un grupo social y susceptible de ser correcta o erróneamente traducido a otro lenguaje».

Lo que nos proponemos en esta segunda sección del artículo es, partiendo de las principales acepciones que la noción de lenguaje recibe en los diccionarios especializados relativamente recientes, continuar la delimitación, en términos semióticos, de un objeto de estudio que - gracias a su naturaleza inter- y trans-disciplinariaaparece definido como relativamente heterogéneo y diverso. Se trata, evidentemente, de la filosofía del lenguaje, al igual que de la metodología adecuada a este objeto, en relación con el que una serie de delimitaciones han aparecido ya en la primera sección del presente estudio y a las que nos vamos a referir en una serie de estudios ulteriores.

En cuanto a la filosofía del lenguaje, nos basamos, para empezar, en una definición de Emanuel Vasiliu (1984: 56), que dice que:

Si se trata de hablar de compromiso ontológico y si admitimos que ciertos signos denotan grupos, entonces este compromiso no es del semiólogo sino de la colectividad, que utiliza cierto lenguaje [...]. Se puede observar que, planteado en estos términos, el problema se escapa del marco de la semántica (que estudia la relación signo/ denotación) y se sitúa en el ámbito de la filosofia del lenguaje.[s.n., M.N.].

Los matices introducidos más arriba nos parecen pertinentes, sobre todo desde la perspectiva que nos interesa, es decir, la semiótica. Por lo tanto, vamos a revisar de manera crítica y en función de nuestras propias opciones, las acepciones de los términos lengua y lenguaje del Diccionario enciclopédico de semiótica -EDS-(1986: 706-721). Al 
igual que en la primera sección del presente artículo, estas acepciones van a ser matizadas con observaciones complementarias entresacadas de otros trabajos de especialidad.

Según lo demuestran los autores del EDS (1986: 709), «la polisemia del término language [que, del inglés, se puede traducir sea por lengua, sea por lenguaje], se puede expresar bajo la forma de [varias] oposiciones:

1) Lingua verbalis [es decir] la lengua hablada, por ejemplo, el inglés, versus lingua mentalis, es decir «un lenguaje que consiste en las significaciones de las expresiones, donde tratamos las significaciones o bien como unidades psíquicas, o bien como entidades objetivas».

En cuanto a nosotros, optamos por tratar las significaciones como entidades objetivas, la psicología quedando, según lo hemos mostrado, totalmente fuera de nuestra esfera de interés.

Esta primera oposición se basa, evidentemente, en un acercamiento psicológico y/o en uno de los métodos de la semántica lógica. Estas dos esferas del conocimiento se van a situar fuera de nuestras preocupaciones relacionadas con el ámbito en discusión.

2) Según la segunda acepción, el término poli-semántico language implica la oposición «lengua versus habla; esta oposición está incluida en la discriminación hecha por Saussure entre langue, langage y parole».

No podemos estar en desacuerdo con las observaciones arriba mencionadas, aunque hay que precisar, como lo han hecho, de hecho, muchos investigadores (Greimas-Courtès, 1979, 1986; Colapietro, 1993) y se ha convertido hace mucho tiempo en aserto, que semejante concepción binaria lleva a una semiología (a menudo productiva, como lo es la de Greimas o como la glosemática) y no a una semiótica.

En cuanto a nosotros, consideramos semejante concepción sobrepasada.

La perspectiva que nos parece la más adecuada para el estudio teórico del lenguaje y de las disciplinas conexas, como la lingüística general y la filosofía del lenguaje, es la perspectiva de Peirce (Net, 1985, 1991, 1992a, 1992b, 1994, 1995, 1997a, 1997b). 
En este sentido, hay que mencionar, también, una observación de E. Coseriu, que aceptamos completamente: «Para ambas ciencias [la lingüística general y la filosofía del lenguaje], las lenguas y los actos lingüísticos no son más que el material ejemplificador, pero desde puntos de vista diferentes» (Coşeriu 1995: 19-20).

También compartimos la opinión de Floarea Vîrban (en un estudio que va a aparecer), que sigue en la misma línea. La autora considera que: «Mientras que la filosofía del lenguaje se basa, en lo subsidiario, en una concepción filosófica que constituye el paradigma dentro del cual se refiere a los hechos concretos de lengua, sin llegar a su descripción o su sistematización, la linguística parte precisamente de estos fenómenos concretos y trata de construir un sistema que ofrezca una descripción general del lenguaje».

En cuanto a la pareja saussuriana langue/parole, preferimos la pareja, de origen chomskyano, competencia vs. performancia (Chomsky, 1967), aunque, tal y como los especialistas lo han subrayado muchas veces (por ejemplo, Colapietro, 1993), la equivalencia entre las dos parejas de términos está lejos de ser perfecta. Añadimos que, en nuestra opinión, la filosofía del lenguaje se ocupa (o debería hacerlo) también de la competencia y de la performancia.

Consideramos sin embargo que la semiótica de Peirce va a tener que ser permanentemente respaldada por los estudios dedicados a la filosofía del lenguaje por la teoría francesa de la enunciación (KerbratOrecchioni, 1980; Ducrot, 1984; Moeschler-Reboul [1994] 1999), la mayoría de origen bajtiano (Bajtín [1963] 1970).

Semejante perspectiva implica un acercamiento pragmático, en la línea de Charles Morris (1938), pero incluye también el análisis del diálogo y de la conversación (véase, por ejemplo, Langages 1969, $1970,1983,1984)$, basados, como es sabido, en la teoría de los actos de lenguaje, tal y como ésta ha sido esbozada por Austin (1961) y Searle (1969). En cuanto a los actos de lenguaje, desde la perspectiva que aquí nos interesa, no debemos olvidar la observación de $A-Z$ ([1994] 1999: 300), según la cual «tienen condiciones de cumplimiento imperceptibles, que pueden desnaturalizar nuestro discurso o pueden generar un fracaso de nuestras acciones. De aquí proviene el interés por este tipo de acercamientos pragmáticos al lenguaje».

Es evidente que, en nuestra opinión, todas estas disciplinas de la lingüística tienen (cada una por separado y todas en común) un componente de filosofía del lenguaje. 
3) Según la tercera acepción del término language del EDS, éste implica la oposición «lengua natural $v s$. lenguaje artificial, por ejemplo, esperanto».

Precisamos que este aspecto está fuera de nuestra esfera de interés.

Añadimos que hemos traducido el sintagma artificial language del inglés por «lengua artificial», debido a la referencia, en contexto inmediato, a la lengua esperanto.

Artificial language se puede traducir, sin embargo, también por «lenguaje artificial».

La distinción entre «lengua natural» y «lenguajes artificiales» está presente también en Sémiotique (1979: 205), cuyos autores especifican que: «lo que llamamos lengua natural se opone, según todas las apariencias, a los lenguajes "artificiales", en cuanto que es algo característico de la "la naturaleza humana" y, al mismo tiempo, trascendiendo a los individuos que la utilizan; se presenta como una organización estructural inmanente, que domina a los sujetos hablantes; incapaces de cambiarla, aun cuando sean capaces de construir y manipular lenguajes artificiales».

Hay que añadir que, bien que se trate de una lengua artificial (esperanto) o de un lenguaje artificial — por ejemplo, el alfabeto Morse, el alfabeto Braille, el alfabeto de los sordo-mudos o «el lenguaje informático», del que habla $A-Z$ ([1994] 1999: 296-299)—, el científico debe tener una visión coherente (aunque sólo, a menudo, implícita) de la relación entre res y verbum, para lograr crear un lenguaje artificial. Tampoco nos va a interesar este aspecto en los estudios ulteriores.

4) En conformidad con la cuarta acepción del termino language del $E D S$, el concepto que discutimos aquí cubre también la oposición entre «lenguaje formal», es decir el lenguaje falto de interpretación (sentido) y «lenguaje no-formal».

Semejante distinción debe ser matizada, en nuestra opinión. Consideramos, por ejemplo, discutible el hecho de que los lenguajes formales (o formalizados, cf. infra) deban ser (necesariamente, por su naturaleza) faltos de sentido. Más bien compartimos la idea de la teoría glosemática (Hjelmslev, 1953), según la cual el sentido es una sustancia seleccionada por una forma, lo que hace que ninguna de estas dos categorías pueda existir independientemente una de la otra. 
5) En este punto, el EDS llama la atención sobre el hecho de que la noción de language cubre al mismo tiempo la oposición entre el lenguaje formalizado - como es, por ejemplo, el lenguaje de un sistema deductivo, compuesto por símbolos primitivos (términos primitivos) y el lenguaje no-formalizado.

En los estudios de filosofía del lenguaje en vía de realización, el aspecto arriba mencionado nos va a preocupar exclusivamente como soporte para una meditación referente al metalenguaje.

Los autores del Diccionario Sémiotique (1979: 209) dicen, en este sentido, que «es, prácticamente, imposible trazar la frontera entre lo lingüístico y lo metalingüístico, [porque podemos considerar] todo discurso científico [por consiguiente también la filosofía del lenguaje, n.n., M.N.], toda ciencia, como de naturaleza metalingüística».

Por nuestra parte, consideramos que hay que tener siempre en cuenta y ser objetos de un estudio aparte las diferencias entre el metalenguaje (literario o no) y la filosofía del lenguaje.

6) El EDS distingue, igualmente, entre «lenguaje en sentido lógico, es decir, un lenguaje que pone de manifiesto una correspondencia de 1/1 entre una expresión y su contenido [el lenguaje de la informática, arriba mencionado se podría encuadrar también en esta categoría], y lenguaje en sentido lingüístico, es decir, una multitud, en sentido lógico [o, más bien, en sentido lógico-matemático] de lenguajes, que contiene[n], por lo tanto, expresiones poli-semánticas, al igual que sinónimos».

Se impone afirmar que el primero de los términos de la equivalencia es una creación artificial, y creemos que ha sido postulada en esta relación más bien para evidenciar las virtudes del segundo término. Añadimos que este último también puede ser a menudo (por lo menos parcialmente) creado. Nos referimos, por supuesto, al lenguaje poético o incluso al lenguaje cotidiano con valencias poéticas. Y estas últimas pueden entrar, según nuestra opinión, en la esfera de preocupaciones de la filosofía del lenguaje.

7) En el penúltimo punto, el $E D S$ incluye la oposición entre lenguas étnicas y lenguas no-étnicas.

Estas diferencias deben, por supuesto, explicitarse, cosa que los autores del $E D S$ omiten. Creemos que aquí también entrarían, de hecho, posiblemente como subdivisión, en una edición ulterior del $E D S$, las diferencias entre norma y uso, entre el lenguaje estándar y el cotidiano, entre la lengua literaria, por un lado e idiolecto, sociolecto, 
dialecto, etc., por otro lado. En nuestra opinión, las distinciones de este tipo tampoco serían ajenas a las preocupaciones de la filosofía del lenguaje.

8) En el último punto, los autores del EDS precisan que existe una oposición entre «las diferentes lenguas, consideradas por separado y la lengua, en general —una idealización y una abstracción-».

No podemos no compartir semejante observación. Sin embargo, es necesario añadir que, desde la perspectiva de esta oposición, debemos discutir también las relaciones entre enunciación, discurso y enunciado (Ducrot, 1984), por lo tanto las reglas para poner la enunciación en el discurso y para materializar este último en enunciado.

Además, se dice a continuación en el diccionario citado (1996: 709), que «el termino language se aplica, sin restricciones, a los diferentes sistemas de signos que no pueden ser considerados lenguajes en el sentido más estricto del término; por ejemplo, a la multitud de síntomas que indica que a un coche se le está agotando la batería».

Se incluye aquí un primer sistema de' señalización, sistema formalizado, parecido a los de la matemática o de la física. Con la diferencia de que a estos últimos se les puede asociar - gracias a un metalenguaje riguroso y explícito- una concepción sobre el mundo, aunque implícita. En esta situación no se puede hablar ya de síntomas, ni de sistemas primarios de señalización. Todo lo contrario, se vuelve evidente la correlación entre las leyes de la naturaleza (o las de la física), por un lado, y la filosofía, por otro lado. Esta problemática le ha preocupado a H.-R. Patapievici (1996: 43-92) en un estudio teórico bastante reciente.

Desde la misma perspectiva, hay que recordar que en el $A-Z$ ([1994] 1999: 300) se precisa que el lenguaje no es sólo un sistema de signos que sirve a la comunicación de ideas o a la representación del mundo, sino que «es, en igual medida - y además-, en el mayor grado, una actividad social. Esta dimensión pragmática del lenguaje ha dado lugar a toda una corriente filosófica contemporánea». De hecho, se nos dice en la misma obra ([1994] 1999: 209) y con razón, que: «hoy se insiste sobre todo en el carácter histórico y social del lenguaje, que no puede ser concebido sin un aprendizaje progresivo y tampoco fuera de todo contexto cultural».

Es una opinión que compartimos. Añadimos, a pesar de todo eso, que no hay que prestar menos atención a una de las principales con- 
clusiones a la que llegan los autores del EDS (1986: 713) en el capítulo dedicado a la filosofía del lenguaje; conclusión que compartimos por completo:

La lingüística contemporánea [...] tiene un carácter explicativo y teórico mucho más evidente. [...] Por consiguiente, el objeto y los métodos de la lingüística teórica contemporánea se han acercado mucho más al objeto de la filosofía del lenguaje, en cuanto disciplina autónoma de estudio, y a la filosofía del lenguaje, en cuanto método. Este acercamiento es tan importante que, por lo menos a veces, ciertas ramas de la lingüística teórica llevan la etiqueta de filosofia del lenguaje.

A menudo, las interferencias y las superposiciones pueden ser productivas. Es, en nuestra opinión, el caso del fenómeno arriba mencionado.

Como conclusión provisional, constatamos que esta revisión, cerrada circularmente, de las principales acepciones atribuidas al concepto de lenguaje en el EDS —en correlación con los sentidos atribuidos al mismo término en una serie de otros diccionarios relativamente recientes- nos lleva, en general, a opciones metodológicas parecidas a las que llegamos en la primera parte de este artículo, al examinar atentamente las posibles facetas del término filosofía.

Hay que añadir que el análisis minucioso de las acepciones y de las observaciones que implican los términos de lenguaje, filosofía y filosofía del lenguaje en otros diccionarios especializados (Julia [1991] 1996; Flew [1979] 1996), análisis que, para evitar la redundancia excesiva, no hemos reproducido aquí, ha llevado a conclusiones similares.

Podríamos, pues, resumir todo lo anteriormente expuesto de la siguiente manera:

La perspectiva más adecuada para abordar la filosofía del lenguaje nos parece la semiótica de Peirce. Esta disciplina es ella misma (entre otras) - circular, pero sólo aparentemente desde un punto de vista tautológico- no sólo una filosofía del lenguaje, sino también un metalenguaje. A este marco general se le deben sumar la pragmática y la teoría de la enunciación, en calidad de objetos posibles de la filosofía del lenguaje, pero también como métodos particulares (la pragmática no es, según ha precisado Morris hace mucho tiempo, una de los tres componentes básicos de la semiótica: Morris, 1938).

Una segunda observación que se impone se refiere al hecho de que una monografía dedicada a la filosofía del lenguaje va a tener que 
tomar en cuenta los distintos metalenguajes específicos de algunas disciplinas particulares como la poética, la retórica o la sociolingüística.

Por fin, la interdependencia entre la filosofía del lenguaje y el estudio de las mentalidades no se puede dejar de lado.

Todos estos aspectos van a constituir el objeto de unos estudios futuros.

\section{Referencias bibliográficas}

San Agustín (1994). De magistro. Ediție bilingvă. Traducere de Mihai Rădulescu si Constantin Noica. Introducere și note de Lucia Wald. Bucuresti: Humanitas.

Austin, J. L. (1961). Philosophical Papers. Oxford: Oxford UP.

Bajtín, M. ([1963] 1970). Problemele poeticii lui Dostoievski. București: Univers.

BIBLIA SAU SFÎNTA SCRIPTURĂ (1975). . București: Editura Institutului Biblic si de Misiune Ortodoxă al Bisericii Ortodoxe Române.

CARNAP, R. (1960). Meaning and Novelty. Chicago: University of Chicago Press.

Chомsкy, N. (1967). Syntactic Structures. The Hague: Mouton.

- (1986). Knowledge of Language. New York: Praeger.

Clément, E.; Dominique, C.; Hamsen-Love, L. y KahN, P. ([1994] 1999). Filozofia de la A la Z. Bucuresti: ALL.

Colapietro, V. M. (1993). Glossary of Semiotics. New York: Praeger.

COŞERIU, E. (1995). Introducere în lingvistică. Cluj: Echinox.

DuCROT, O. (1984). Le dit et le dire. Paris: Minuit.

FLEw, A. ([1979] 1996). Dictionar de filozofie și logică. București: Humanitas.

Greimas, A. J. y CourTès J. (1979). Sémiotique. Dictionnaire raisonné de la théorie du langage. Paris: Hachette.

- (eds). (1986). Sémiotique. Dictionnaire raisonné de la théorie du langage. Paris: Hachette.

HiNTIKKA, J. (1969). Knowledge and Belief. An Introduction to the Logic of the Two Notions. Ithaca \& London (fourth printing): Cornell UP.

HJElmsLev, L. (1953). Prolegomena to A Theory of Language. En Memoir to the International Journal of American Linguistics, supplement to volume XIX, 1, Baltimore.

JuLIA, D. ([1991] 1996). Dictionar de filosofie. Bucuresti: Univers Enciclopedic. Kalish, D. y MONTAGUE, R. (1964). Logic Techniques of Forward Reasoning. New York: Harcourt, Bruce and World, Inc. 
Langages 13, 1969: L'analyse du discours.

Langages 17, 1970: L'énonciation.

Langages 70, 1983: La mise en discours.

Langages 73, 1984: Les plans de l'énonciation.

Kerbrat-Orecchioni, C. (1990). L'énonciation. De la subjectivité dans le langage. Paris: Armand Colin.

Moeschler, H. y Reboul, A. ([1994] 1999). Dicționar de pragmatică. Cluj: Echinox.

MORRIS, Ch. (1938). Foundations of the Theory of Signs. Chicago: Chicago UP.

Net, M. (1985). «A Poetic Mask to Peircean Semiosis». Revue Roumaine de Linguistique (RRL) 4, 341-350.

- (1989). Metalimbajul textului literar. București: Tipografia Universității din Bucuresti.

- (1991). «Types d'interprétants du texte poétique». Angewandte Semiotik. Linguistik Interdisziplinär II, 35-46.

- (1992a). «Etapes de la sémiose peircienne». En Signs of Humanity/L'homme et ses signes, 1311-1317. Berlin: Mouton de Gruyter.

- (1992b). «A Few Remarks on Socio-Cultural Symbols». En Semiotics 1990 \& Symbolicity. Part II, 134-139. Lantham, MD: University Press of America.

- (1994). «Mentalities and Cultural Interpretants». S - European Journal for Semiotic Studies 3-4, 675-690.

- (1995). «La lexicologie dans la perspective de la sémiotique peircienne». Semiotică si poetică 6, 5-19.

- (1997a). «Clichés and Empire-Builders». En Fonctions du cliché. Du banal à la violence, 71-79. Tours: Presses Universitaires de Tours.

- (1997b). «On Cultural Stereotypes. A Peircean View». En Semiotics Around the World. Synthesis in Diversity 303-315. Berlin/New York: Mouton de Gruyter.

Patapievici, H. R. (1996). «A Theory of the Internal Medium». En New Europe College Yearbook 1994. București: Humanitas \& New Europe College.

Platón (1989). Opere, vol. III. Traducere de Simona Noica. București: Editura stiint ifică si enciclopedică.

Searle, J. R. (1969). Speech Acts. Cambridge: Cambridge UP.

SEBEOK, Th. A. (1981). The Play of Musement. Bloomington: Indiana UP.

- (General Editor). (1986). Encyclopedic Dictionary of Semiotics, vols. I-III. Berlin: Mouton de Gruyter.

VASILIU, E. (1984). Sens, adevăr analitic, cunoaștere. București: Editura științifică și enciclopedică.

VîRBAN, F. (2001). «Preliminarii la o analiză fenomenologică a limbajului». Studii și cercetări lingvistice (SCL) 1 (en prensa). 